\title{
SURVIVAL AND PROGNOSTIC FACTORS OF BREAST CANCER IN WOMEN IN THE STATE OF SÃO PAULO
}

Raissa Janine de Almeida1, Carolina Terra de Moraes Luizaga², Cristiane Murta-Nascimento

${ }^{1}$ Faculdade de Medicina de Botucatu, Universidade Estadual Paulista - Botucatu (SP), Brazil.

${ }^{2}$ Fundação Oncocentro de São Paulo - São Paulo (SP), Brazil.

Introduction: Breast cancer is the first most common malignancy in the female population worldwide. Monitoring the survival of women with breast cancer has been a strategy frequently adopted at the international level as a measure to assess the progress of public policies for the control of the disease. Objectives: To estimate the probabilities of five and ten year survival and to investigate the prognostic factors of women with breast cancer included in the hospital-based cancer registry (RHC) of Fundação Oncocentro de São Paulo (FOSP). Methods: It was a historical cohort study. The sample consisted of women with breast cancer diagnosed between 2002 and 2012 and included in the RHC-FOSP. The event of interest was breast cancer-specific mortality. Living cases at the end of follow-up (December 31, 2017), loss to follow-up, and death other than that due to breast cancer were considered censored on the date of the last contact or date of death. Descriptive analysis and survival analysis were performed using the Kaplan-Meyer method. Survival curves were compared using the log-rank test. Hazard ratios (HR) and their 95\%CI were estimated using Cox’s proportional hazards model. This study was approved by the Human Research Ethics Committee of the Botucatu Medical School, São Paulo State University, Brazil. Results: Between 2002 and 2012, 53,146 cases of invasive breast cancer were registered at RHC-FOSP. The median age of women at diagnosis was 55.9 years. By the end of the follow-up, 20,683 patients died and $71.4 \%$ of such deaths were due to breast cancer. The five and ten year breast cancer-specific survival for the entire cohort was 76.1\% (95\%CI 75.7-76.5) and 64.8\% (95\%CI 64.2-65.3), respectively. In the multivariate analysis, the factors associated with prognosis were: age at diagnosis, year of diagnosis, educational level, grouped clinical stage, and histological type. Conclusions: Specific survival for breast cancer in the state of São Paulo is significantly associated with several characteristics. The knowledge of these characteristics can contribute to the development of public policies in the area. 